Review article

\title{
LASER SCANNING IN ENGINEERING SURVEYING: METHODS OF MEASUREMENT AND MODELING OF STRUCTURES
}

\author{
Grzegorz Lenda, Andrzej Uznański, Michał Strach, Paulina Lewińska
}

AGH University of Science and Technology, Faculty of Mining Surveying and Environmental Engineering, Department of Engineering Surveying and Civil

Engineering, Krakow, Poland

\begin{abstract}
The study is devoted to the uses of laser scanning in the field of engineering surveying. It is currently one of the main trends of research which is developed at the Department of Engineering Surveying and Civil Engineering at the Faculty of Mining Surveying and Environmental Engineering of AGH University of Science and Technology in Krakow. They mainly relate to the issues associated with tower and shell structures, infrastructure of rail routes, or development of digital elevation models for a wide range of applications. These issues often require the use of a variety of scanning techniques (stationary, mobile), but the differences also regard the planning of measurement stations and methods of merging point clouds. Significant differences appear during the analysis of point clouds, especially when modeling objects. Analysis of the selected parameters is already possible basing on ad hoc measurements carried out on a point cloud. However, only the construction of three-dimensional models provides complete information about the shape of structures, allows to perform the analysis in any place and reduces the amount of the stored data. Some structures can be modeled in the form of simple axes, sections, or solids, for others it becomes necessary to create sophisticated models of surfaces, depicting local deformations. The examples selected for the study allow to assess the scope of measurement and office work for a variety of uses related to the issue set forth in the title of this study. Additionally, the latest, forward-looking technology was presented - laser scanning performed from Unmanned Aerial Vehicles (drones). Currently, it is basically in the prototype phase, but it might be expected to make a significant progress in numerous applications in the field of engineering surveying.
\end{abstract}

Keywords: engineering surveying, terrestrial laser scanning, mobile laser scanning, UAV-borne laser scanning 


\section{Introduction}

Over the last several years, laser scanning has become one of the most important methods of acquiring information about structures which are of interest to engineering surveying. Its main advantage, compared to other methods, is the possibility to obtain a full model of the structure, and not just its selected geometrical parameters. The result of scanning is a point cloud, constituting the basis for obtaining a 3D model, followed by its characteristic elements such axes, edges, cross sections, surface areas, etc. Sometimes the modeling of the entire structure is not required, or sometimes it is too complex, and then it can be reduced to approximation of the desired elements of the structure only, or read selected information directly from the cloud. Regardless of the final use and the type, a continuous model of the structure, replacing a discrete, dense point cloud, allows for a considerable reduction in the amount of the data needed to be stored, and facilitates a comparison of periodic measurements.

This paper presents the uses of terrestrial laser scanning in the field of engineering surveying and the related applications. The main issue relates to measurement methodologies and also provides examples of data modeling. The objects which were selected were of various size, structure and specific character: elongated and surface structures, with regular and irregular shape. Elongated structures can be both uniform objects, for example tower structures, as well as extensive investments related to communication routes. In this case, scanning methods (stationary or mobile), the methods of data modeling, and the obtained geometric parameters subject to assessment, may be different. Surface structures can be characterized by a diversified scale as well: from the shell of a shell structure, to extensive digital elevation models. Also in these cases, it is possible to use not only stationary, or, more broadly, terrestrial scanning, but the combined use of scanning and the UAV technique is increasingly gaining significance, which can be used, for example, to determine the cubic volume of bulk materials. Depending on the shape of the surface (regular or not) and the required accuracy of the models, analysis of the data may be performed based on various approximation methods.

The study discusses examples involving the measurements of industrial chimneys, shell structures, infrastructure related to railway routes and elements of the digital elevation model. A separate section is devoted to the UAV scanning, which is currently treated as a tool in its testing stage, but with a considerable potential to be used in the field of engineering surveying.

Research studies on these issues are currently being conducted at the Department of Engineering Surveying and Civil Engineering at the Faculty of Mining Surveying and Environmental Engineering of AGH University of Science and Technology in Krakow.

\section{Tower structure on the example of an industrial chimney}

Industrial chimneys are examples of tower structures, for which one of the key parameters determined during as-built measurements is the shape of the vertical axis. It is most frequently determined using the method of bisector directions tangent to the surface, or the polar method (Gocał, 2010), observing at least a few selected levels of the structure. The result of the study is the axis in the form of a broken line, related to the selected levels, presented in projections to all planes of the coordinate system. The measurement and calculation procedures are simple and fast, however, 
they lack other information about the geometry of the building structure. Laser scanning, providing information which is sufficient to build a 3D model of the whole structure, allows primarily to determine the precise, curvilinear shape of the axis. It also enables to identify strains, losses or cracks of the chimney shaft. A considerable advantage of the scanning technique is a short observation time of the proper construction, which is favorable due to its temporary strains associated with insolation and wind pressure.

The essential elements of the measurement procedure include the appropriate selection of observation stations and adjustment points for merging scans. These activities are more stringent than for classical measurement methods, and the difference derives from the object of observation. In order to obtain a full point cloud representing the structure, it is required to ensure visibility of its entire surface from the scanner positions. Building structures adjacent to the chimney make the selection of scanning positions difficult. They should be stabilized right at the walls of the buildings (Fig. 1.b), and sometimes achieving a complete visibility is impossible (Fig. 1.c), which affects the subsequent modeling of the structure on the basis of an incomplete point cloud.

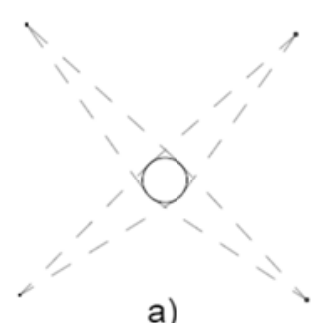

a)

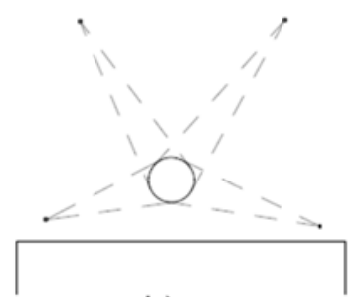

b)

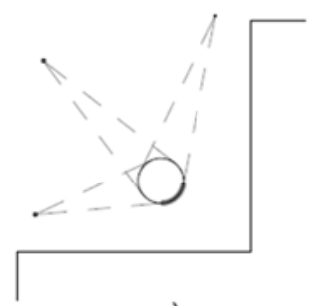

c)

Fig. 1. Arrangement of observation stands for the measurement of a chimney using laser scanning. The part of the structure which was invisible during scanning has been marked with a bold line

For the classical methods of measurement, three stations are optimal, whereas for scanning it is worth increasing their number to four. Reflectorless measurements carried out at very acute angles, virtually along the tangents to the shaft, frequently do not produce any result; it is also vitiated by major errors. The size of the areas at the edges of the structure which these problems relate to, may be subject to a number of factors (Lenda et al., 2015), such as the type of the scanner (including its range, carrier wavelength), the type of the chimney surface (metallic and synthetic surfaces increase these areas in comparison to brick surfaces), temporary changes in reflectivity of the surface associated with weather conditions (heat, humidity), the color of the structure. If the point clouds obtained at adjacent positions have the planned mutual coverage which is very limited, it may turn out that the merged point cloud, having eliminated the outliers, has significant gaps in its structure. Such a situation may occur when measurements are performed from only three stands. With increasing their number to four stands, a sufficient margin of the mutual coverage of adjacent clouds is ensured.

Another reason for increasing the number of measurement stands regards merging the clouds into a uniform coordinate system. Generally, tower structures have surface too small to ensure good merging accuracy known as cloud to cloud, however, it may be a method supporting the standard merging using reflective targets. The scanning of building structures carried out from only three stations may provide insufficient coverage of the neighboring clouds, or it will occur for the points 
with low accuracy. The coverage is to some extent dependent on the range of the scanner - the greater it is, the farther away the stands may be arranged from the chimney, which consequently increases the range.

The range is a key factor determining the applicability of the scanning technique for the measurements of tower structures of significant height. It is definitely reduced by soot and other pollutants, but red and white warning stripes located in the upper parts of chimneys may cause a problem as well. A laser beam with a green dot (e.g. Leica ScanStation C10) is strongly absorbed by the red color of the surface, resulting in every other stripe being poorly measurable (Fig. 2.a). Scanning of higher chimneys most frequently requires a significant approach of the measurement stands towards the structure, and measurements of their upper parts are carried out at unfavorable, steep lines of sight.

Point clouds obtained from adjacent positions are usually merged by means of separately scanned adjustment points (reflective targets or spheres). Their arrangement should form an irregular spatial solid with the span comparable to the size of the structure which, in the case of tower structures, may be difficult to meet. Due to the industrial infrastructure surrounding chimneys, to ensure visibility from the neighboring stands, these points are generally established at a short distance from the positions of the scanner, which can be located in close proximity to the chimney. The area where the points are located, usually has a smaller (often significantly smaller) horizontal span than the height of the observed structure. Additionally, vertical span of the target grid, conditioned by the possibility to access high structures, is usually limited to a few meters. From the perspective of the scale of a tower structure, the arrangement of reference points can therefore be regarded as close to coplanar, with a smaller span than the dimensions of the structure. Such geometry results in the errors of the elevation coordinate of the target inducing several times greater error of fitting clouds at great heights. Therefore, it is particularly important to ensure a stable arrangement of targets. To strengthen their spatial structure, it is advisable to additionally use the points located on the higher parts of the chimney, which can be distinguished on adjacent scans. These fragments should be scanned separately, with high resolution to get the best fitting of the clouds. The scanning from four observation stands, as it was mentioned above, contributes to the appropriate coverage of the clouds.

Filtration of point clouds, which allows to eliminate these observations which are not the chimney construction (galleries, ladders, antennas, lighting, etc.), is an important issue preceding the process of the proper modeling. Generally, this process is carried out manually, but it is possible to use M-estimation algorithms (Huber, 2009), removing outliers from a given model by a predetermined value. This model can be a cylinder, a cone, or a set of planes (in the case of octagonal brick chimneys). Automatic filtering effect can be assessed in Figure 2.a. The final filtering of the cloud is always carried out manually, though, which prevents the removal of the points illustrating local deformations of the structure.

Analysis of the results, depending on the required information, leads to the formation of a regular (ideal solid), irregular (surface taking into account deformations of the structure), and cross-sectional model. The regular model, as for the filtration of the cloud, is created by using elementary geometrical solids or groups of solids. It allows to acquire information about local deformations of the structure with respect to its design shape, by determining the distance between the points and the surface (Fig. 2.b). The presented model contains points with larger deviations $(12-30 \mathrm{~mm})$ resulting from inaccurate filtering of the points from exterior installations. However, 
the ideal mathematical model in the form of solids does not allow to determine the shape of the chimney axis. Only the irregular model offers such a possibility, presented in the form of the Triangulated Irregular Network (TIN) (Li et al., 2004) or approximating spline surfaces (Kiciak, 2005). Triangulated Irregular Networks are usually created using Delaunay triangulation. For a dense cloud of points they ensure a good approximation of the shape of the chimney. The density may, however, be significantly reduced by the factors illustrated in Figure 2.a: great losses in point clouds associated with poor reflection of the beam from the red warning belts, and those related to the target being obscured with exterior installations. This may impair the information read from the model created by flat triangles with a significant span. The most advanced method for the modeling of a chimney surface are approximating spline functions (Fig. 2.c). They allow to create a surface with continuous curvature, whose tension reduces local losses in the point cloud. This type of a model may be used to carry out periodic comparisons of changes in the shape of the shell and to determine the continuous curvilinear image of the vertical axis. Such an image is formed by the algorithms determining the medial axis (Siddiqi et al., 2008) based on the left and right horizontal curve of the chimney in a suitable cutting plane (Fig. 2.d). In some cases, there is no need to create three-dimensional models of the whole structure, it is enough to develop selected horizontal or vertical sections. To determine them, slices of point clouds are used, which have a certain thickness, usually not more than of a few centimeters. This is necessary to ensure an adequate density of the observation along the section line, which may then lbe created based on regular models (circles, straight lines) or irregular models (splines).

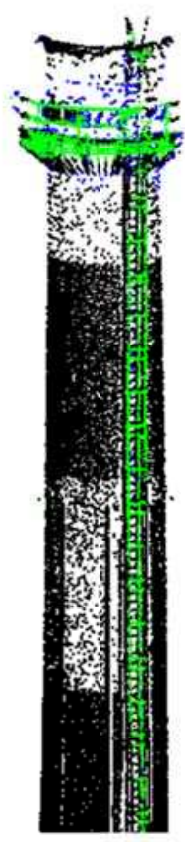

a)

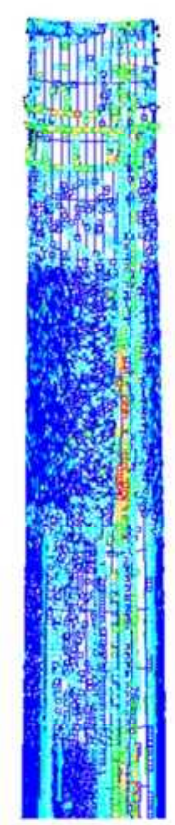

b)

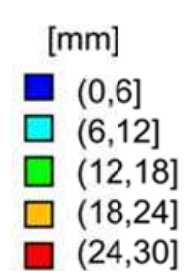

Fig. 2. Examples of developing models based on the data from laser scanning: a) result of automatic filtering of exterior installations, b) deviations of a point cloud from a regular solid (a cone), c) approximating spline surface, d) medial axis determined based on the vertical section of the spline surface

The discussed problems remain valid also for slender structures other than chimneys, with considerable elongation in the vertical direction (columns, poles, etc.). 


\section{Shell structure on the example of a multi-patch roof}

Scanning measurements of shell structures become a standard tool for obtaining geometric information about the objects of this type. Unlike the tower structures, for which the shape of the axis is important, in the case of shells, the most important information is about the shape of the shell surface. Classical methods of measurement (angular spatial intersection method and polar method using reflectorless total stations) are very time-consuming, and moreover, the first one requires targeting the existing or determined points. The resulting set of observations of small size, typically not exceeding a few hundred points, does not sufficiently reflect local deformations of the shell.

Laser scanning seems to be the currently the most appropriate tool for measurements of this type of structures. The point cloud and the models created on its basis, offer the possibility of a detailed analysis of local shell deformations. The general principles for the arrangement of measurement stations and targets in the case of some shells which are also tower structures at the same time (e.g. cooling towers), are similar to those for chimney measurements. For the observations of shells, there is usually a higher number of scanner stands, which results from a much larger span of the structure in the horizontal direction, and often its level of detail (Fig. 3 ). The key factor in the selection of the stations is the range of the scanner, especially due to the greater variety of materials and colors that can cover the shell. Scanning of the object presented in Figure 3, due to its complex structure which consisted of seven surface patches of different size, required the use of six measurement stations. In the case of shells of a significant spread in the horizontal direction, the selection of targets is less critical. Even if it is not possible to install them at a greater height, the large area which they cover is protected from failure to fit the upper parts of the clouds by the merging process. In addition, when merging adjacent scans, due to a large surface being observed, the cloud to cloud method could be used as a support. A necessary condition for this method is obviously an adequate margin of the cloud coverage, and good merging accuracy is supported by high scanning resolution. In particular, the process of merging all the scans can be performed only basing on the cloud to cloud method, which significantly accelerates the measurement, eliminating the part related to the arrangement and scanning of the targets. If merging of the clouds is carried out with their use, neighboring stations should be merged by at least three targets. Most frequently, they are arranged on the structure or in its close vicinity, which makes a specific point visible only from two, or at most three, adjacent stands. For comparison, with a favorable arrangement of targets during measurements of the chimney, it is possible to see the same points from all positions of the scanner (only four such points may prove to be sufficient). For the measurement of the structure illustrated in Figure 3.a, 19 targets were used, which gives an idea of the amount of work associated first with their arrangement, and then scanning. As a result of the measurements, the cloud visible in Figure 3.b. was obtained. It has gaps in the observations related to the structure being obscured by the elements such as vegetation, additional installations on the structure, or adjacent surface patches.

Just as for the tower structures, point cloud must be filtered to eliminate installations covering the surface. Automatic algorithms are of limited use here, because the surface patches are not necessarily simple geometric solids, in relation to which the process of eliminating the outliers can be carried out. In the case of the structure discussed here, the observations inside the building structure, near the 
walls, which were created by the beam passing through glass patches, proved especially time-consuming and difficult to filter.

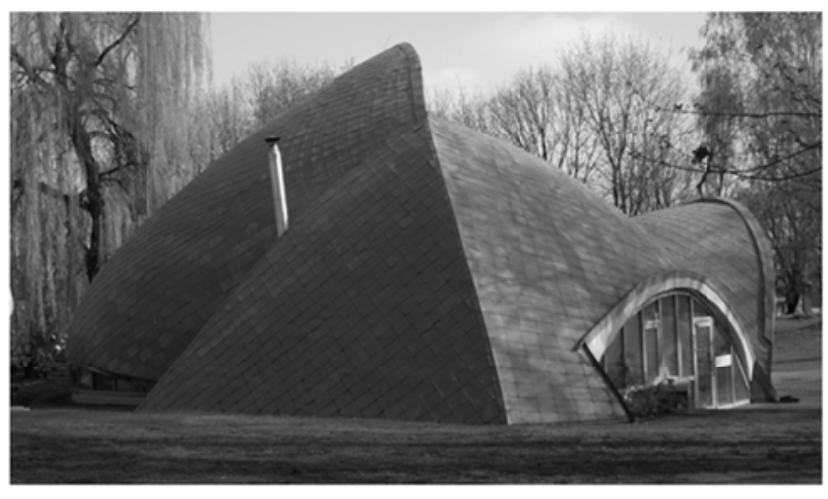

a)

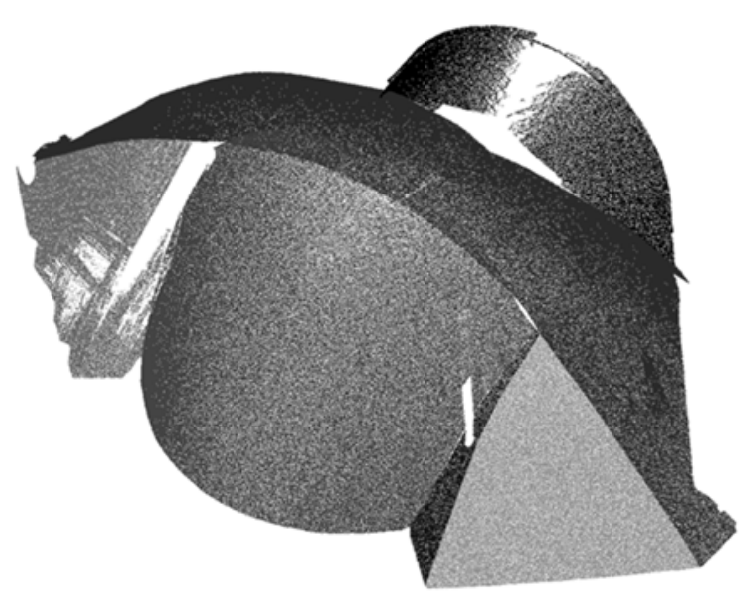

b)

Fig. 3. Seven-patched shell structure: a) general view, b) point cloud divided into individual patches and gaps in observations caused by the structure being obscured locally

Modeling of the shape of shell structures should be performed using techniques which allow for the description of local shell deformations. Such a possibility is provided by the two previously mentioned methods: Triangulated Irregular Network and approximating spline surfaces. TINs represent a model which is locally linear, not preferred in the studies of the shell shape with a continuous curvatture, especially at the places with decreased point cloud density. The spline functions ensure such a continuity. Until recently, their use for the modeling of data from laser scanning was not possible, due to the lack of appropriate software. Available applications (Rhinoceros, Maya, 3D Studio Max) allowed only for the modeling of interpolation spline surfaces, rigorously passing through the measured points. Such a surface is formed based on all the points from the merging of the patches, each of which is extended on four corner points. Calculation capacity of modern computers is far from sufficient to achieve such a task, especially that the spline surfaces are determined in a sequential process, which means the lack of opportunity for multicore calculations. Approximating spline surfaces allow to significantly reduce the number of generated surface patches, thanks to the additional use of the method of least squares. The extent of each patch is determined arbitrarily, as a result of a preliminary analysis of the geometry of the point cloud. Then, it is fitted into a group of points of any size, limited only by the range of the patch. At the same time, the process of merging adjacent patches with continuity of curvature is performed. With this solution, a good compromise is achieved between fast calculations and fitting the surface to the point cloud, which is on the average level of several millimeters. This type of modeling allows to make overlays on standard graphic packages, for example: RhinoResurf or RhinoReverse.

The first one was used to build the model of the discussed, seven-patch shell structure. For each of the patches, separate approximating spline surfaces were formed, which were ultimately merged into one model along the edge lines (Fig. 4.a). Figure 4.b illustrates the analysis of the accuracy of fitting the shell into a point cloud.

As a result of modeling, the average accuracy of fitting was achieved at the level of $2.1 \mathrm{~mm}$. Larger deviations, $9-15 \mathrm{~mm}$, appeared locally, and were mainly due to the 
deformations of sheet metal plates covering the whole object. Part of the deviations (right side of the structure) arises from the difficulty in filtering the observations inside the object. Some of the points, measured through glass at acute angles (which contributes to the occurrence of erroneous observations), were found between the inner and outer shell of the structure. Some of them were so close to the modeled outer shell that they were not rejected in the filtration process, deforming the spline surface. The upper part of Figure 4.b illustrates the arched belt of large deviations $(12-15 \mathrm{~mm})$. It was created by modeling using one spline surface both the patch and the adjacent rim, between which there was a several-centimetter offset. Spline functions do not provide a good approximation of parts with discontinuous curvature, which is manifested by larger deviations, but thanks to them it is possible to identify such places easily. In this case, the rim should be modeled by a separate surface.

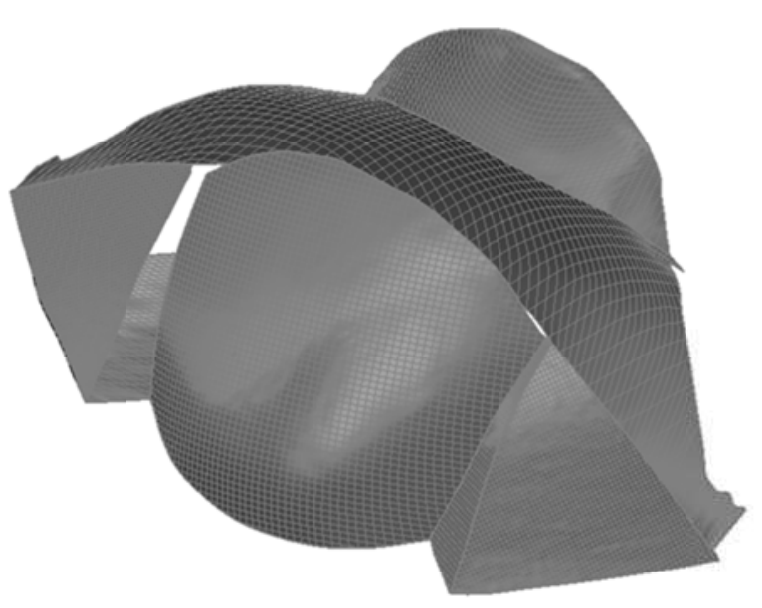

a)

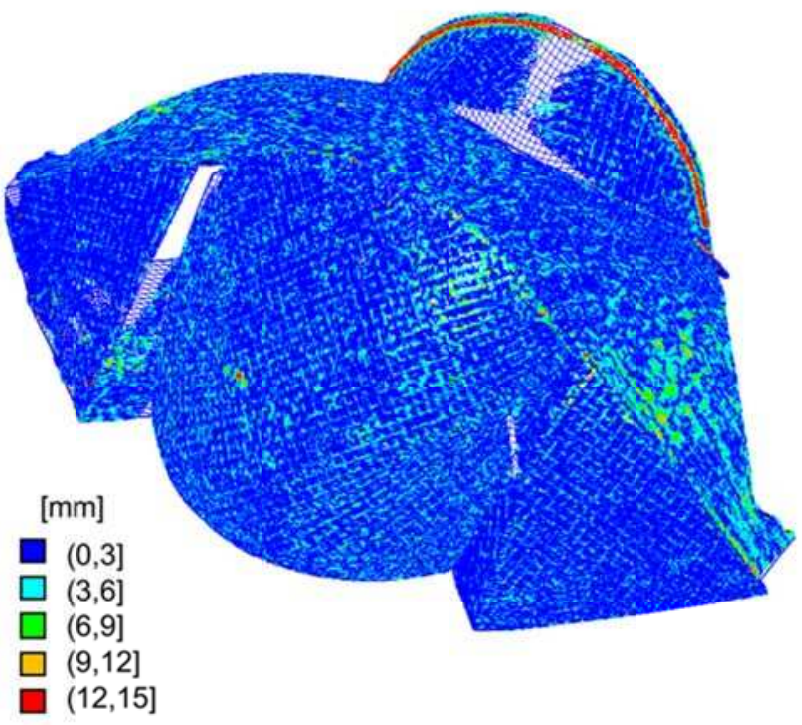

b)

Fig. 4. a) approximating spline model of the structure, b) analysis of the accuracy of fitting the model into a point cloud

Similar analyses can be used to determine the differences relative to the design model and during periodic measurements to illustrate changes in the shape of the shell over time.

\section{Analysis of the technical condition and geometry of tunnels and rail transport infrastructure}

In some measurements of building structures and technical infrastructure, the time required to complete a given study, and the costs associated with it, play an important role. There are also works in which access to the surveyed structure is limited, which is dictated by a large distance, lack of visibility (obscuring, no lighting), or security reasons (power cables, structure in use). These problems occur during the surveys of linear structures, which include for example: transport routes, tunnels, public utilities. Surveying of these structures can be carried out lby the commonly used, classical measurement techniques. They are being increasingly replaced by terrestrial laser scanning in the stationary and mobile versions. 
In the construction of the mobile laser scanning systems (MMS/MLS), two types of solutions are identified. The first one are flexible systems. In this case, any configuration of individual measurement devices is allowed, with the use of stationary 3D scanners and 2D profile scanners. However, systems dedicated to mobile measurements are used more often. They use profile scanners integrated with the positioning devices GNSS/IMU. Regardless of the selected solution, the MMS/MLS system consists of the following components:

- laser scanner - stationary or profile;

- positioning system, consisting of: GNSS receiver and IMU inertial unit;

- odometer (DMI);

- set of photogrammetric cameras or video cameras;

- on-board computer;

- other optional components (e.g. GPR).

The system for mobile laser scanning is installed on the vehicles traveling on the road and/or rail route at speeds from a few to more than a hundred kilometers per hour. The system is usually installed on the roof (Fig. 5a), or on various platforms (Fig. 5.b-c) and columns (Fig. 5.d), which the vehicles are equipped with.

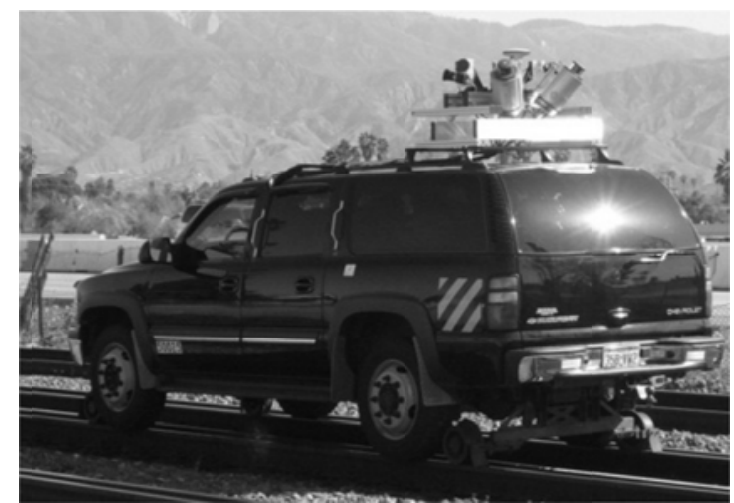

a)

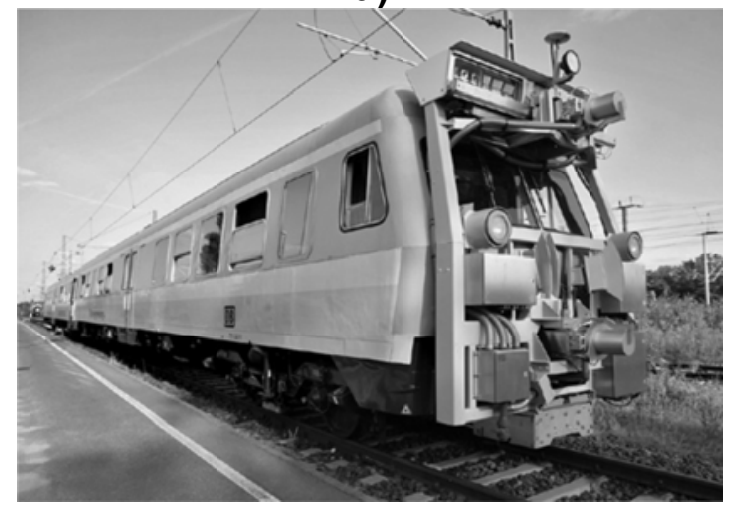

c)

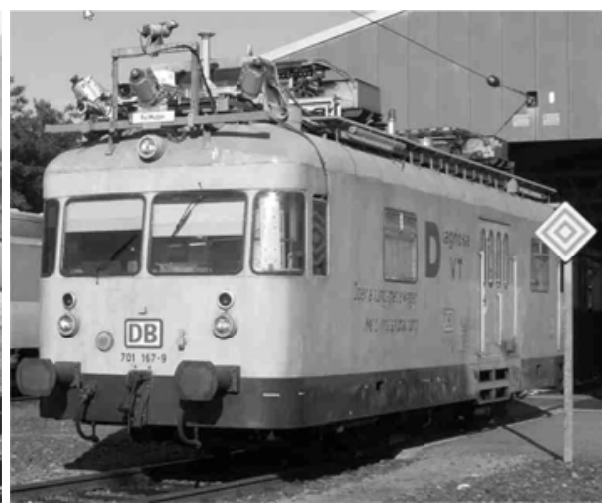

b)

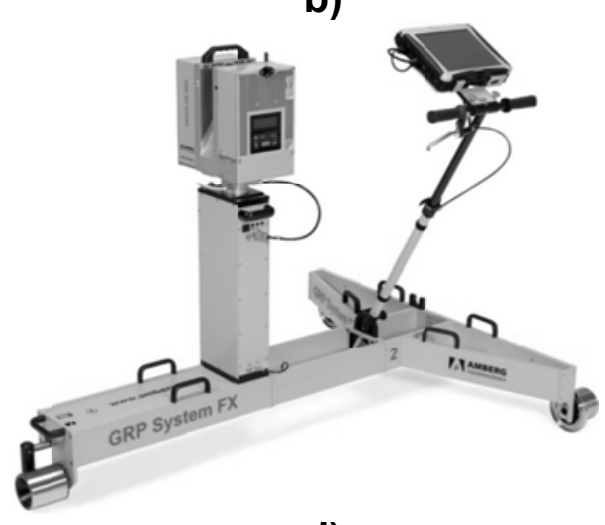

d)

Fig. 5. Examples of mobile laser scanning systems for railway applications: a) Street Mapper and b) Rail Mapper by IGI and 3D Laser Mapping (www.streetmapper.net, 2016);

c) LIMEZ III working for German Railways (Wirth, 2008); d) GRP 5000 Rail by Amberg Technologies (www.ambergtechnologies.ch, 2016).

Each of the components of the MMS/MLS system, records observations in its own frame of reference. In order to merge them and calculate the coordinates of the scanned points, a series of transformations should be carried out, and the observations ought to be merged using the time stamp. In the process of system 
calibration, offsets between its individual devices are determined. The reference point is usually the Inertial Measurement Unit. In the case of derdicated systems, integrating various components, calibration is the manufacturer's responsibility, who provides the user with specific offset values. In flexible systems, the user has to perform the calibration. Regardless of the applied solution, it is required to regularly check the calibration parameters and, if necessary, make appropriate adjustments.

One of the applications of laser scanning is assessment of the technical condition and the geometry of tunnels, which require periodic surveys (Hoult, Soga, 2014). Due to poor lighting and limited space, tunnels are structures which are difficult to survey. Another problem, affecting the safety of work, can be vehicular traffic. Depending on the length of the structure and the type of communication, appropriate scanning methods are used (Gikas, 2012). For the tunnels whose length does not exceed a few hundred meters, stationary laser scanning may be used (Fig. 6). For those whose length exceeds several kilometers, mobile scanning is recommended, due to the fast rate in data recording.

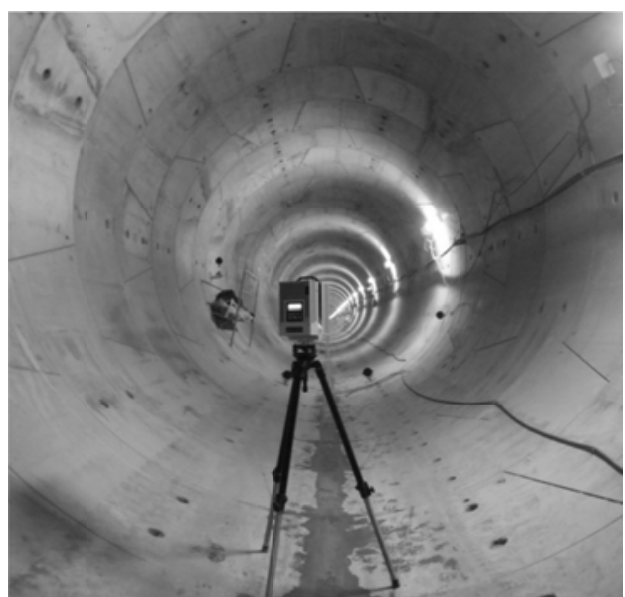

a)

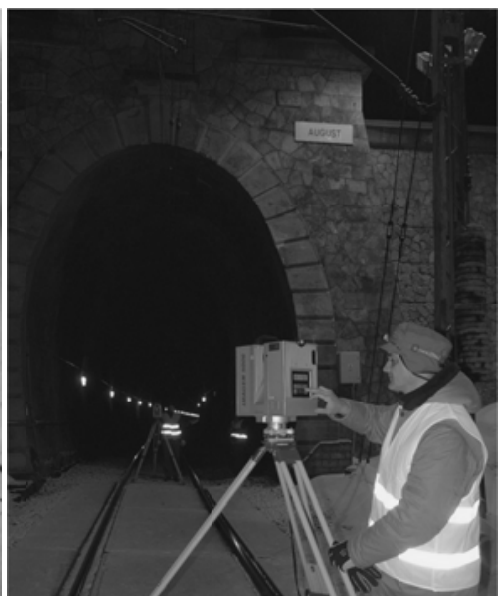

b)

Fig. 6. Terrestrial laser scanning in the tunnel: a) the second line of the Warsaw subway, b) on the railway line No. 8 near the "Tunel" railway station.

The use of mobile scanning technique in the measurements of road and railway tunnels involves considerable limitations, especially with regard to the limited availability of the GNSS signal. The problem is reflected in the obtained absolute accuracies in determining the position of the measuring system. In the case of a prolonged lack of signal, the accuracy can drop even up to tens of meters. To solve this problem, appropriately dense arrangement of photogrammetric control points and the high-end IMU and DMI devices included in the positioning system are used. The registered point cloud could be a source material for the preparation of as-built documentation, as well as the basis for the conformity assessment of the executed structure and the design (Fig. 7) (Zhang et al., 2013). It is also possible to analyze the technical condition of the structure and the geometry of the tunnel lining (Pejić, 2013). The fragment of the point cloud representing the longitudinal section of the inner lining of the tunnel (Fig. 8), shows the elements which characterize its design and technical condition. The identifiable elements include: cracking (1), expansion joint (2), zones of water penetration (3). Also stone blocks (4) are visible, which make up lining of a part of the tunnel (4), as well as numerous points arranged along parallel vertical lines (5). These probably are the elements of the anchoring which 
reinforces the lining. The Figure also clearly demonstrates a wrinkled area on the roof of the tunnel (6). Constant distances between the folds of similar characteristics may indicate the use of a special structure in this place, connecting the old and the new section of the tunnel with two different cross-sections.

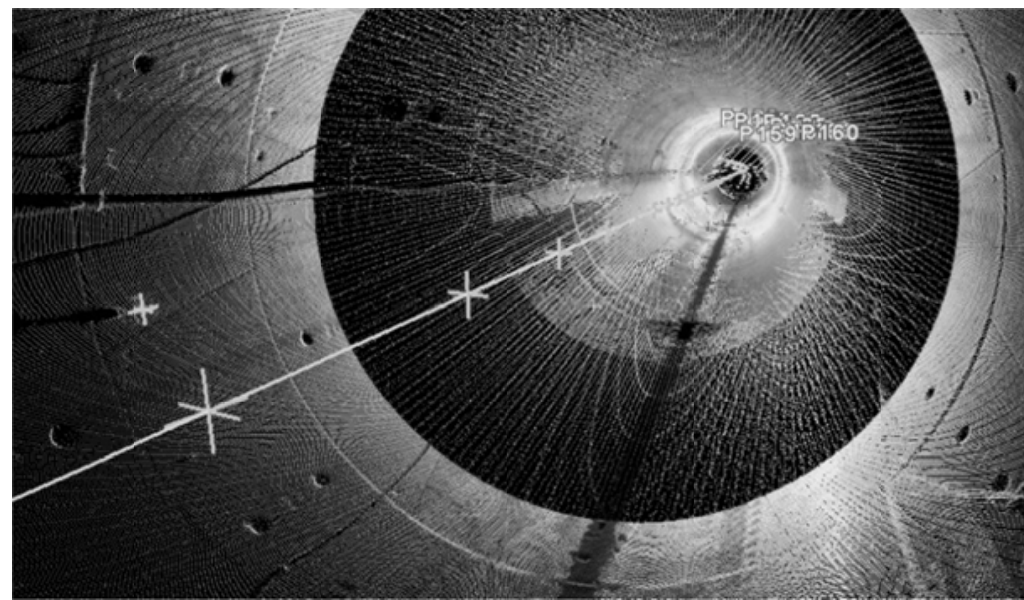

a)

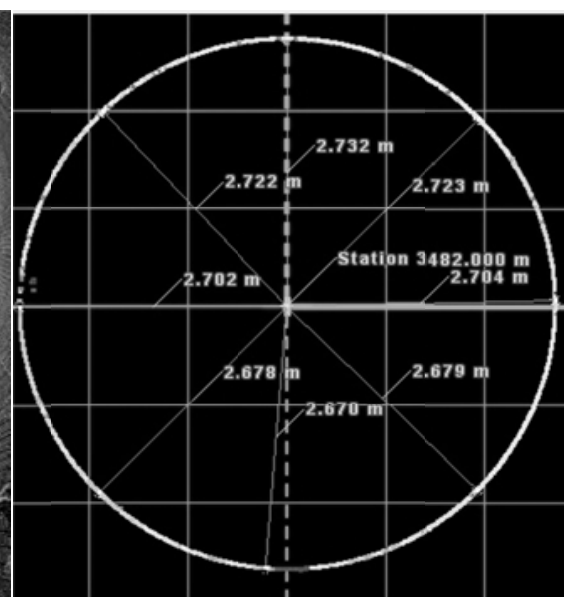

b)

Fig. 7. a) The main axis in the subway tunnel against the point cloud, b) cross section of the tunnel with the measured radii.

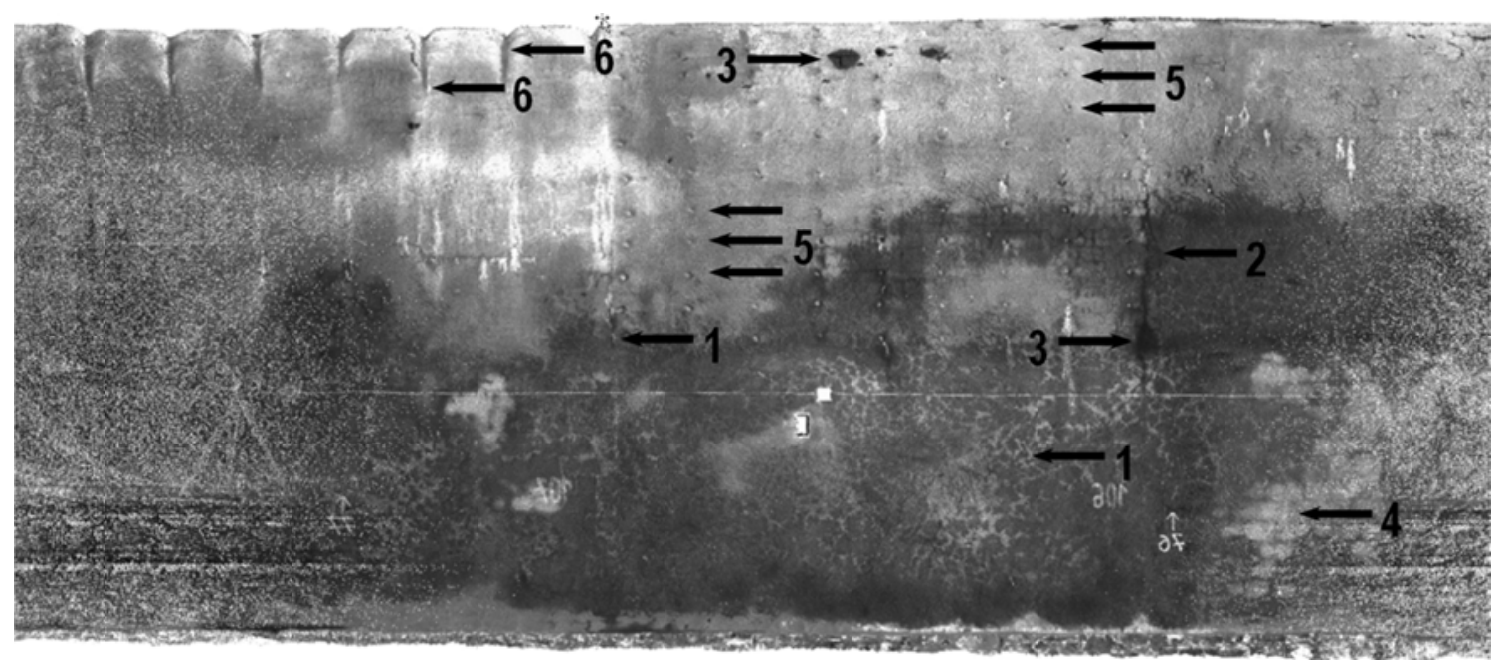

Fig. 8. A fragment of a point cloud representing a longitudinal section of the inner tunnel lining, presented on the basis of the intensity of reflection.

Another, often performed study, is the analysis of the preservation of the clearance gauge (Novaković et al., 2014). This condition must be met on all transport routes, regardless of the used means of transport. Most frequently, however, it is verified on rail routes. The analyses must be preceded by an identification of the current course of the route axis. In the case of railways, first of all, the rolling surfaces and the inner edges of the rail heads must be recognized (Fig. 9.a-b). On this basis, gauge and cant of the track can be calculated (Fig. 9.c). In the middle of the section defining the track width, the position of the track axis is calculated. In the course of the works on identifying the axis of the track, it is possible to use procedures that automate the identification of the spatial position of each of the rails. Most frequently, these are algorithms which analyze the local differences in elevations (Yang, Fang, 2014). These methods have their weaknesses manifested by the problems with the correct 
classification of the points. This takes place in the vicinity of railway traffic control devices, on the sections with check-rails, and at the turnouts. In such cases, an additional step in verification of the classification is used. It is based on data filtration by fitting a rail model.

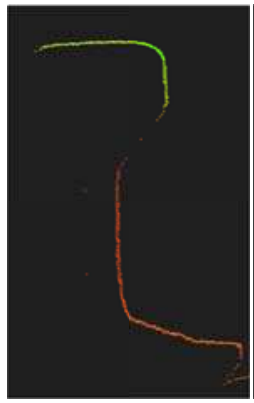

a)

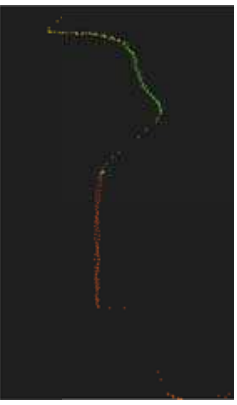

b)

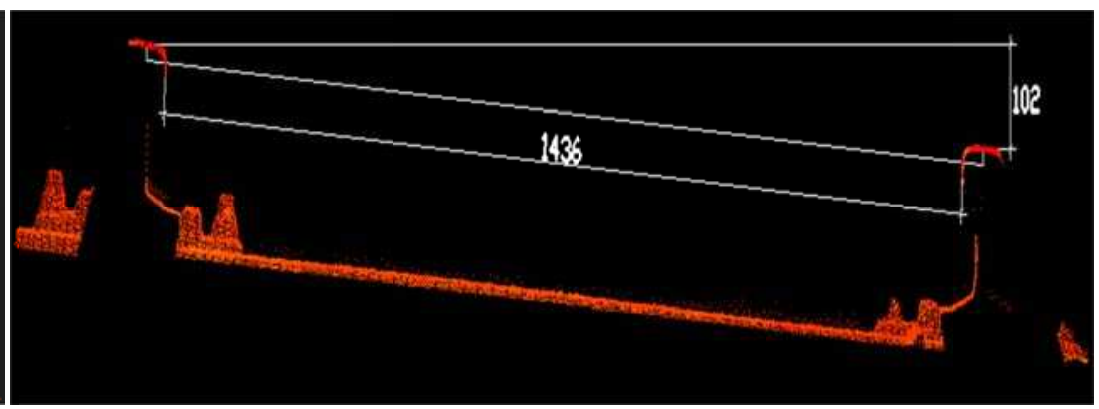

c)

Fig. 9. Cross-sections of a rail: a) correct and b) with visible side wear; c) cross section of the track, with the gauge and cant.

After a proper identification of the position of railheads, and having determined the spatial course of the track axis, analysis of the axis geometry and its position relative to other elements can be performed. The basis for the control of a safe distance between the railroad and the technical infrastructure is the verification of the clearance gauge preservation. For control purposes, a template (outline) of the clearance gauge should be built. The template is applied perpendicular to the track axis, leaning on the rails (Fig. 10). Verification of the clearance gauge preservation can be implemented in two ways. In one method, the template is virtually moved along the track in the 3D point cloud. The user decides at which point (mileage) the template is set and the measurements of the distance between the clearance envelope and the elements of the point cloud are performed. In the second method, cross-sections at the predetermined distances on the track must be generated (Weixing et al., 2014). Then, after applying a template of the clearance gauge, its preservation is analyzed. Exemplary cases of verifying the preservation of the clearance gauge have been illustrated in Figure 10, with the distinction between railway tunnel (Fig. 10 a-b) and railway route (Fig. $10 \mathrm{c}$ ).

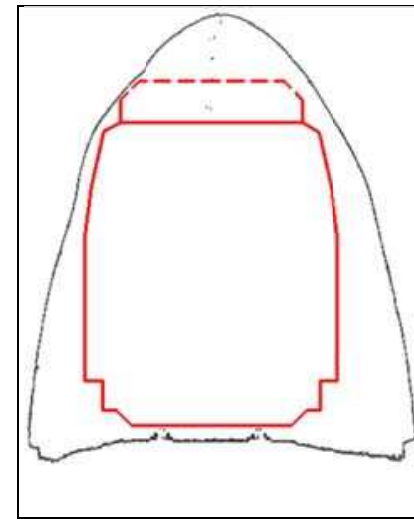

a)

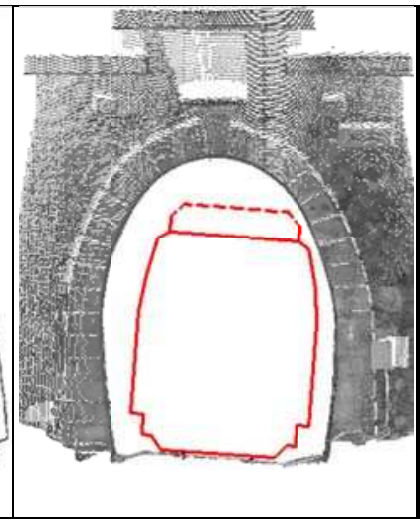

b)

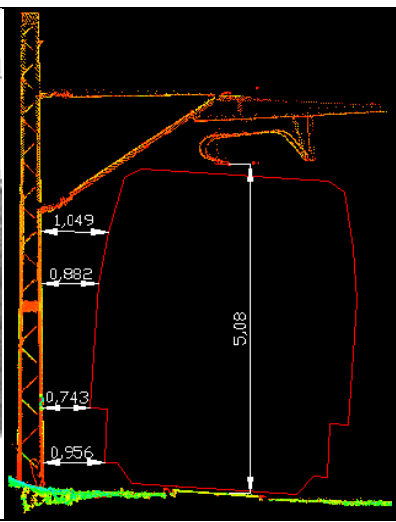

c)

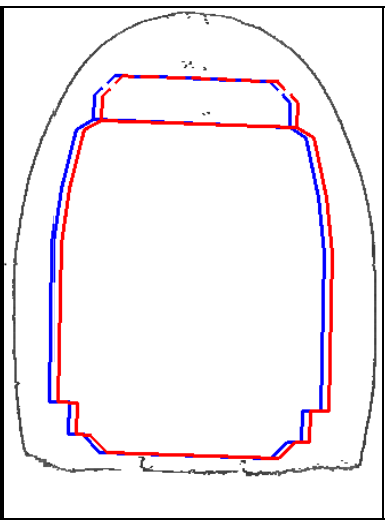

d)

Fig. 10. Selected cross-sections with the clearance envelope: $a, b$ ) in a railway tunnel; c) on the route; d) clearance envelope before and after the completion of the track axis adjustment project. 
For the modernization purposes of the railway tunnel and the railway inside it, track axis adjustment project was prepared (Strach, 2013). The project specified adjustments to the spatial position of the track axis. The project can be implemented under the condition of the preservation of the structure clearance gauge for the new track position in the tunnel. The examination of the clearance gauge has been demonstrated in Fig. 10.d. The envelope of the clearance gauge for the existing track position (red) and designed track position (blue) was plotted on the transverse profile generated from the point cloud.

\section{Laser scanning of topographic objects}

Multiple examples of buildings and other types of manmade structures surveys ware given. Laser scanning has proven to be extremely effective by obtaining vast amount of details in a very short time. The next logical step was to use this technology for surveying of topographical structures.

Laser scanning can be used in monitoring changes in volumes of spoil tips. It allows to acquire fast information on how the size of a tip alters, and also makes it possible to monitor the changes in the angle of slopes on sides of such objects. In order to maintain safety, the slopes must ensure that not even heavy rain is going to make upper layer to slide. Laser scanning is more useful than standard GPS profiling due to obtaining more data in shorter time. However, since most tips are undergoing land rehabilitation process consisting usually of planting various kinds of trees, it is important to plan the survey in advanced, and sometimes it is sufficient to use airborne laser scanning techniques and later filter the vegetation out of the point cloud.

Perhaps the most common usage of this technology is inventory of open pit mines. Excavation in open pit mines is usually done in steps, involving blasting material, transporting it to the preparation plants and then setting explosives for the next blast. Constant survey of the blasting and excavating process must be done. Depending on law and economic reasons, this is being done in various intervals of time, usually weekly to monthly. GPS techniques ware commonly used for profiling the open pit. In cases safety reason prohibit from entering the object UAV-Based Photogrammetry technique is implemented. Those processes, however, is either time consuming or not very accurate. In (Tong et al., 2015) authors are taking into consideration classical methods and laser scanning. A laser scanner like UAV does not need to enter the measured object (the exception being target placement). Also the accuracy is similar to GPS. What is more, laser scanning could be used as reference for cheaper and faster photogrammetry method providing extra reference points.

Other kind of objects that might require higher accuracy during measurements are landslides (Strurzengger et al., 2007; Lenda et al., 2016). Since landslides can be unpredictable, it might be important to measure even smallest, but continuous changes to the observed areas. The result does not necessary need to be a dense point cloud but the clouds done in time intervals need to be in the same coordinates systems. Only then it might be possible to observe small changes.

Laser scanning is a part of remote sensing technology for a reason, it allows for gathering spatial information without accessing the measured structure. This is extremely helpful in regard to measuring objects like open pit mines or cliffs. Setting the stations in appropriate distance from each other and from the object might cause some problems. Taking into consideration the strength of the beam and the density parameters of the used scanners is crucial. With top parts of spoil tips, beach, even 
parts of landslides, there is little chance of placing scanner outside the object that would cover the area in question. In this case, when placing the scanner on the object, the survey staff is left with two problems. One involves setting up many scan stations. Single station, even with a long range scanner when there is no enough elevation, is going to give little information since every higher object provides a 'shade' covering space behind it. The other problem might be the station itself. Scanner has a blind spot underneath where there is no point cloud. This might cause problems during interpolation and later on measuring change in volume (Fig.11). Placing the scanner on the object might not only make the survey longer but also give inaccurate results. Sometimes there is a need to observe dailly, or even hourly changes of delicate objects like sandy river banks or snow cover. Changes that occur is such time periods demands a laser scanner with correct accuracy but also a lot of caution upon entering the object. Figures 11 shows a GRID model constructed from point clouds of a sea shore. Besides the inappropriately placed scanner (a circle on the mesh, scanner should not be placed on measured object) footsteps of the survey staff (depth of footsteps - about $3 \mathrm{~cm}$ average) are visible. Since the total difference between the heights of 2 scans was a few centimeters on average, mistakes like that, if not contracted, might bring an error in calculations.

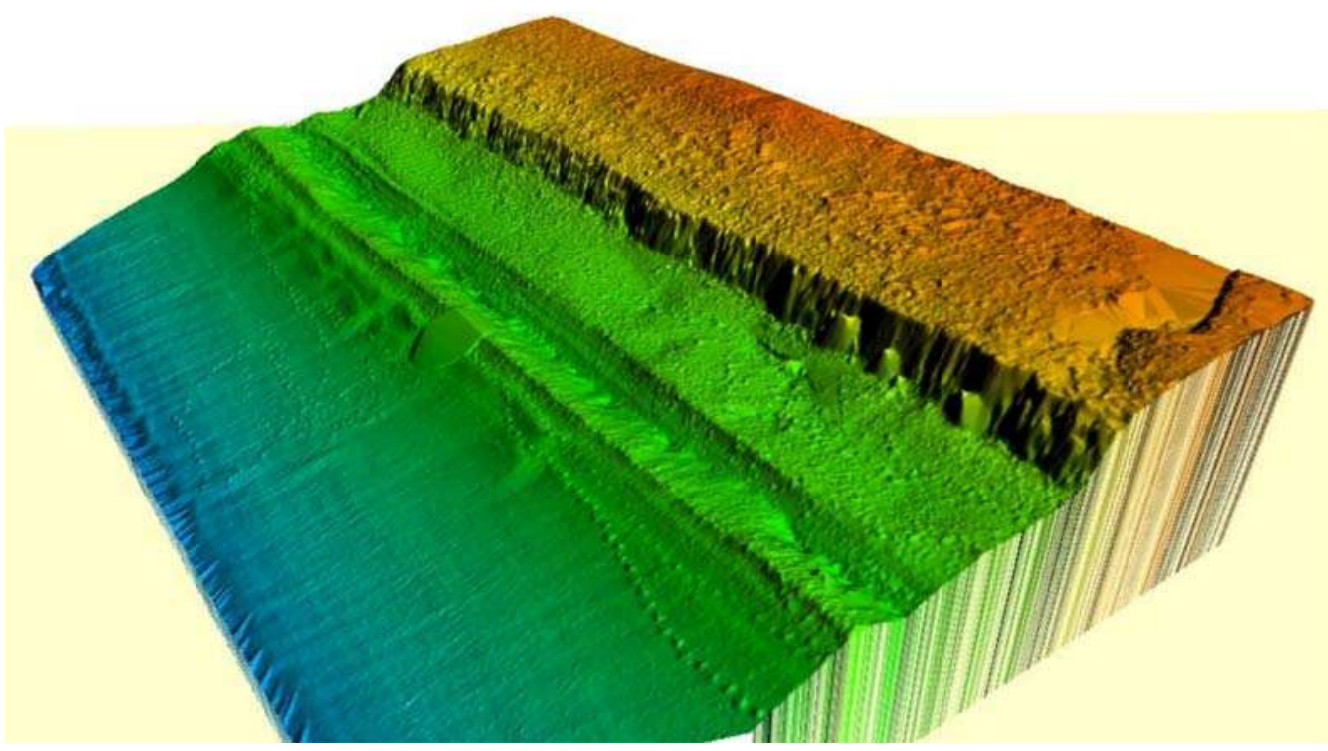

Fig. 11 GRID model of a point cloud of a sea shore: circle represents a blind spot of the scanner, foot prints of survey crew are visible (pic. P. Zagórski)

There are two standard ways of connecting scans. First is the use of targets (chessboards, spheres or other extra objects), placed in the range of the scanners, which software can identify. It can also be done by cloud to cloud method when the software identifies parts of the object as one. The latter method is proven to become more and more popular since it requires shorter survey. The drawback is that cloud to cloud registration of the neighboring clouds needs to have a lot of overlay. This is easily done with buildings, but smaller structures cause problems in the field. It is not always possible to place scanner stations accordingly, for example, plant life safety issues might prevent it. The other issue might be a lack of easily identifiable elements. In this instance it is very important to check the results of software registration. It might show a good accuracy in the report, but often point clouds are 
wrongly placed or rotated, even placed upside down. Sometimes manual registration helps, but the observer not always can spot similar elements.

Point cloud is sometimes viewed as a result of 3D modeling, however, for most practical uses what is needed is a surface or some kind of continuous representation of the object. This can be done in various ways, usually by mesh or interpolation process.

Two basic representations of polygon mesh are known as TIN (Triangulated Irregular Network) or GRID. TIN model is an irregular triangle model that can be obtained either by simply connecting neighboring points with lines that eventually create triangles without any special rules, or by Delaunay triangulation. The second method allows to avoid elongated figures and provides a smoother model. The GRID model consists of a regular field of squares done of points interpolated from original data. Usually it is smoother than TIN model. The usual methods of interpolation are Kriging or Inverse Distance Method (Ries, 1993; Olea, 1997). In more complex software other options like spline interpolation functions are possible (Lenda et al., 2016).

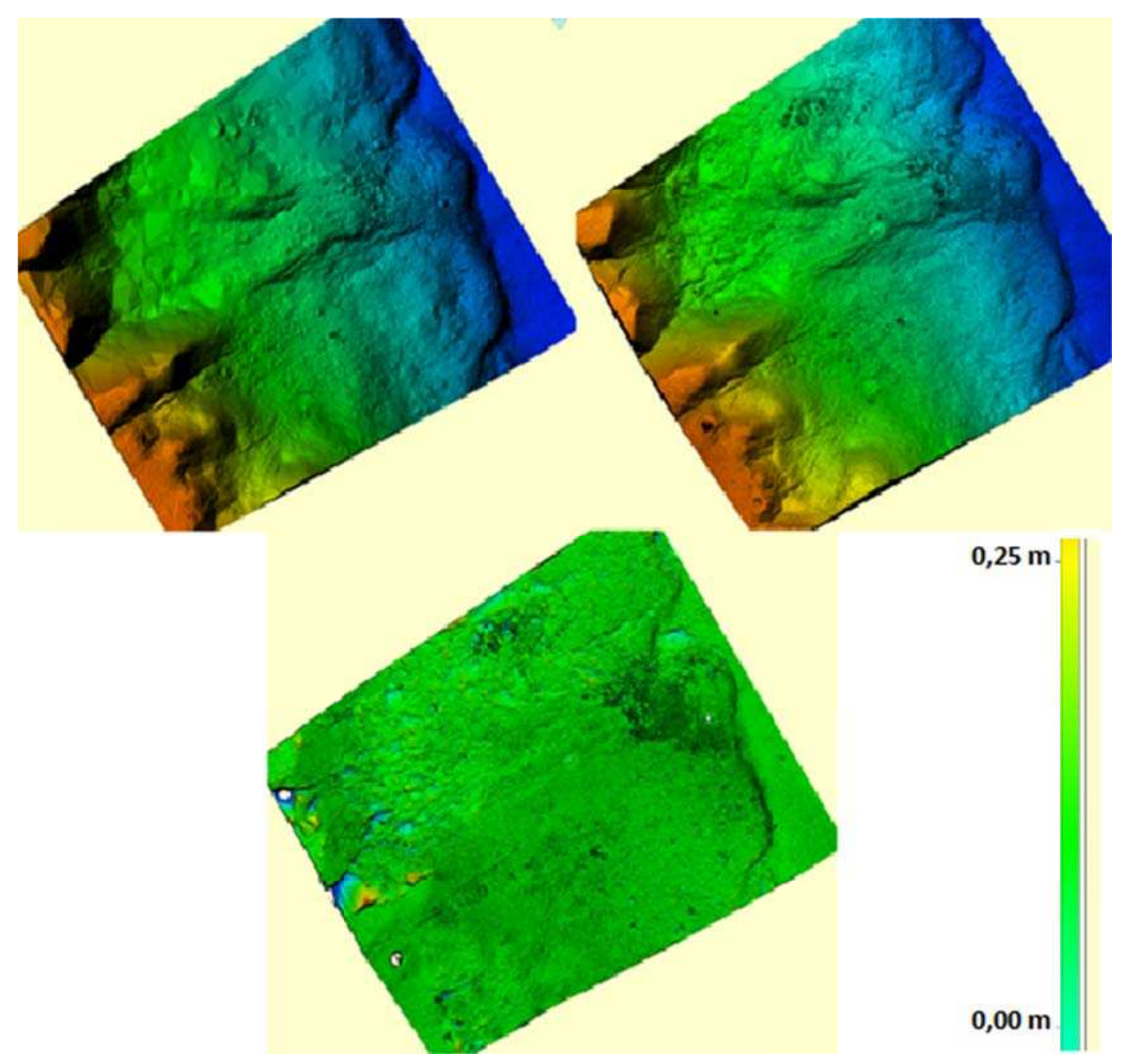

Fig. 12. On the left - TIN model made of $1 \%$ of the original point cloud. On the right - TIN model made of $100 \%$ of the original point cloud. At the bottom - the difference between two models. (Fot. P. Zagórski) 
If a point cloud is dense and the terrain relatively uncomplicated, the means of interpolation are not as important. The difference becomes more apparent if the point cloud is sparse or terrain more complex. In the case illustrated in Figure 12, the difference in height of the slope was about 20 meters. The terrain was a little bit complicated, but there were no greater differences in model between creating a mesh from $1 \%$ and $100 \%$ of original point cloud (fig. 12).

\section{UAV-borne laser scanning}

Laser scanning with the use of UAVs (Unmanned Aerial Vehicles) is a relatively new technique for acquiring information, which is currently developing very intensively. In engineering surveying, this technology should be treated as the one with very good perspectives. For this reason, a decision was made to introduce laser scanning with the use of UAV, although currently it is not used yet in the problems typical of engineering surveying.

From the point of view of the title of this paper, it is important to draw attention to the difference in relation to the most common use of UAVs. Despite the immense popularity and the growing trend of using UAVs for acquiring data, it should be mentioned that in the subject of this study concerning laser scanning, the current use of UAVs is not widespread, nor is it a standard functionality of the UAS (Unmanned Aerial System). The most common use of the UAS is photogrammetry, or acquiring information from photographs taken with a digital camera (CCD camera). The UAS measuring systems, equipped with laser scanners, are currently expensive solutions. The cost is generated not only by the price of ultra-light laser scanner. The installation of such an expensive device with a potentially high-quality measuring results, as a consequence requires the installation of the GNSS (or GPS) receiver and IMU (standard or even tactical class). In such Unmanned Aerial Systems, sometimes one, or even more, digital cameras are installed.

The purpose of the UAS laser scanning is to obtain information about large areas from the air. A particular advantage is the possibility to capture information about inaccessible areas or those which are difficult to access, as well as in the conditions posing a threat to humans. The main innovation contributed by the UAS solutions is the elimination of the remote control, which is necessary in the ALS. This is a very important extension of the availability of data acquisition technology from the air. Another advantage is the significant reduction in project costs due to the replacement of a manned aircraft or helicopter with a much smaller and less expensive UAV. This is not an ideal change, though, and entails limitations for flight conditions. Manned aircraft can fly into the storm. UAVs can fly at wind speeds up to approx. $10 \mathrm{~m} / \mathrm{s}$. On the other hand, the UAV's dimensions allow to use it in places inaccessible to manned aircraft, and provide a possibility to scan from the air while hovering, in the case of multirotor UAVs.

The areas where the UAS laser scanning can be used, depend to some extent on the equipment of the UAV. From the point of view of this study, the minimum equipment of the UAS is a GNSS receiver, IMU, laser scanner, CCD camera. In addition, it is possible to install additional thermal or multispectral imager, which enables a specialized image analysis. An important parameter of each UAV is MTOW (Maximum Take Off Weight) and the associated maximum flight time. Flight time is still a very important drawback of the UAS, because it is usually from a few to tens of minutes. 
The primary application of UAS laser scanning are flights aimed at calculating the volume of landfill materials and earth masses, as well as mime workings. The accuracy of calculating the volume has been studied in (Draeyer et al., 2014), where the results of the UAS photogrametry and LIDAR were comprared. The mean deviation of the surface determined by the two methods was estimated at $2 \mathrm{~cm}$ to 4 $\mathrm{cm}$ (but no reference to a more accurate model was made), and GSD (Ground Sample Distance) was $5 \mathrm{~cm}$. The difference in the determined volumes was in the range of $0.3 \%$ to $0.8 \%$. Due to the inaccessibility of a place and a posed threat to human life, the UAS laser scanning is suitable for the identification and monitoring of landslide areas. In the comprehensive study on the measurement techniques for landslides (Scaioni et al., 2014), the use of the UAS is still presented as a very promising tool for the identification and monitoring of landslide areas. The primary advantage is the ability to conduct flights at very low heights, and consequently, a possibility to obtain a better precision and resolution. In the case of a multirotor UAS, an additional advantage may be precise control of a flight, including hovering. FOV (Field of View) of the UAS laser scanner can reach $330^{\circ}$ or even $360^{\circ}$ and may facilitate the acquisition of a complete set of data during a single flight.
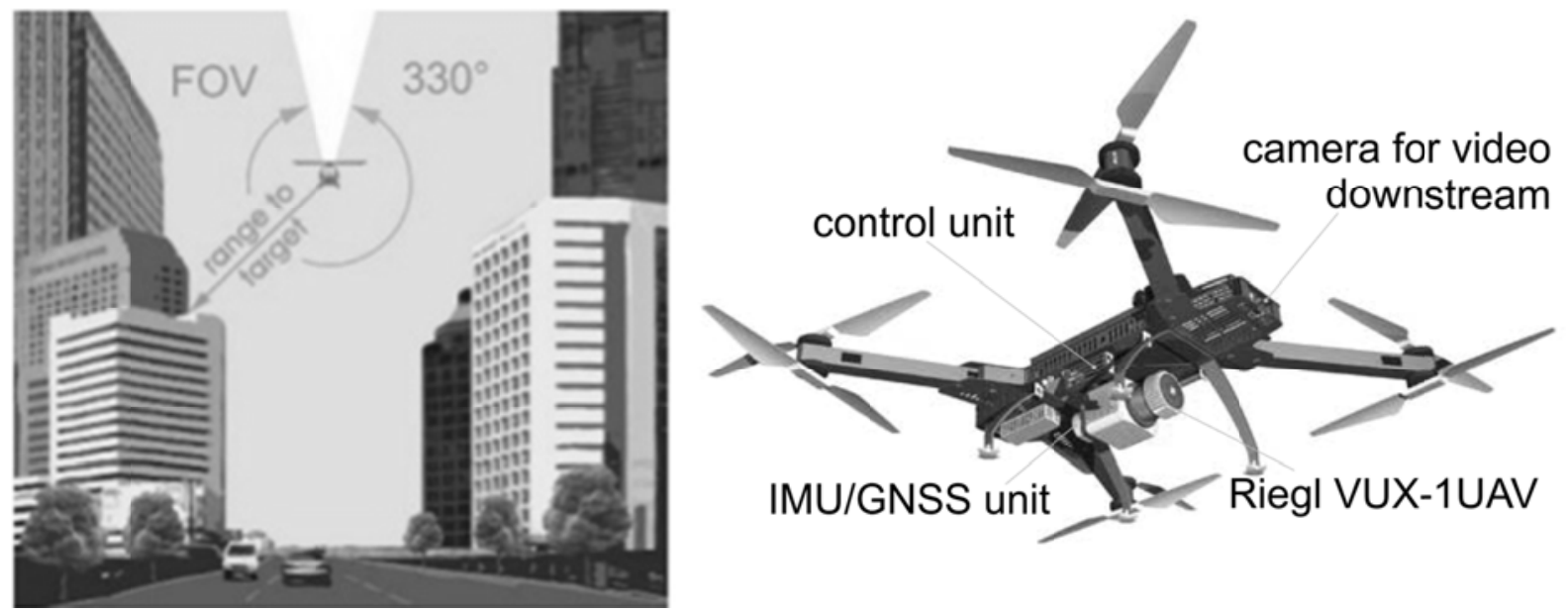

Fig. 13. Field of View Riegl VUX-1 (www.rieglusa.com)

In the case of linear structures, the UAS laser scanning can be used, for example, for road engineering, railway engineering, gas industry, heat engineering, power industry. Surveying, inspection and monitoring of linear structures executed using the UAS can be economically unrivaled in the case of larger structures.

FPV (First Person View) function of the UAS, enabling to detect sites which require special attention in real time, e.g. due to their technical condition, may prove to be useful. Another aspect of practical importance, especially for linear objects, is the competence level of the operator. Undoubtedly, for such applications, the operator with license to fly BVLOS (Behind Visual Line of Sight) has the greatest advantage. No need to follow the UAS, particularly in the terrain which is covered with vegetation, irregular, crossed by rivers or wet, significantly increases the efficiency of measurements performed with the UAS. The UAS makes it possible to assess the technical condition of pipelines, power lines, including the detection of collision, or the assessment of the likelihood of its occurrence with the growing vegetation.

As it was already mentioned, the most widely used element of the UAS is a digital camera. Laser scanning with the use of UAV has the advantage that it can reach the ground surface through vegetation (Lefsky et al., 2002). The importance of this fact 
for the engineering surveying results from the possibility of the measurement signal to reach the structure, which is covered with vegetation.

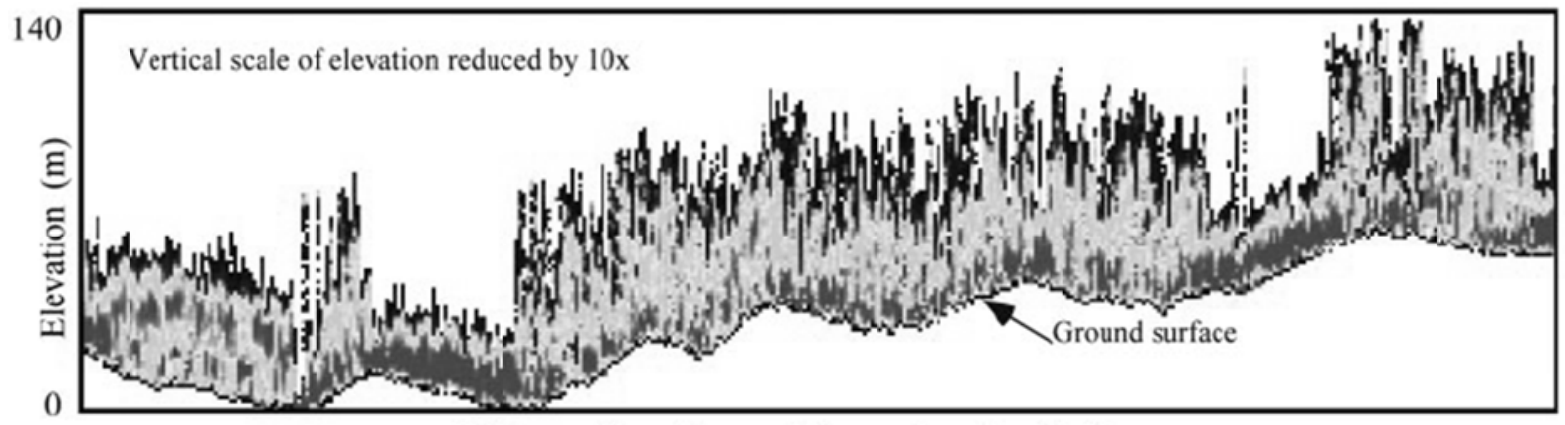

Distance Along Transect (approximately $4 \mathrm{~km}$ )

Fig. 14. Measurements of canopy structure made using NASA's SLICER (Scanning Lidar Imager of Canopies by Echo Recovery) device (Lefsky et al., 2002)

The decisive factor determining the use of the new measurement technique in practice is its accuracy and efficiency. Basing on the source literature, determination of the achievable accuracy of the study results, which use laser scanning with the UAVs, is difficult. Analysis of various studies leads to the conclusion that Unmanned Aerial Systems equipped with a laser scanner, a GNSS receiver, IMU, and a CCD camera, were in most cases only prototyped. The formulation of generally important conclusions about the accuracy of the UAS is difficult because of its prototype character and the usual presence of an element of the quality of results which is substantially lower than those offered by this type of devices available on the market, but unsuitable for the UAS. GNSS models, IMU laser scanners, with their weight adapted to the UAS, are relatively rare. In the analyzed sources, the final result was heavily affected by the quality of the results of the weakest element of the system.

Analysis of the measurement results using the UAV-borne laser scanning are relatively rare in the literature. On the other hand, there are plenty of the study analyses using digital photos from the UAS for different purposes. In order to determine the quality of the results from the UAV-borne laser scanning, the quality of the test results with the use of digital images can be treated as a reference level, on the assumption that the use of the scanner (millimeter distance measurement) will improve the quality of studies, which means that in this case, the accuracy will be higher.

The most reliable method to assess the quality of the results captured from the UAS, is to compare the obtained coordinates with the coordinates of control points. The coordinates as the final effect of the study are analyzed most frequently, but there are also works on the analysis of intermediate stages, for example the accuracy of the trajectory of the moving UAV.

Theoretical analysis of the error sources in LIDAR can be found, for example, in (Glennie, 2007). It also contains a comparison with the values obtained from the test measurements for various equipment configurations.

The study (Nagai et al., 2008) analyzed the quality of the results from the UAS, built of CCD cameras, light laser scanner SICK LMS-291 of the accuracy of $10 \mathrm{~mm}$ for the range of $20 \mathrm{~m}$, GPS Astech G12 receiver and IMU Tamagawa Seiki TA7544. The horizontal accuracy was estimated at $3-6 \mathrm{~cm}$, and the height at $10 \mathrm{~cm}$. Nevertheless, the results were mainly affected by the use of a single-frequency GNSS receiver of poor quality in the system. An additional problem was the 
synchronization of the GNSS receiver with the frequency of $1 \mathrm{~Hz}$ with the IMU with the frequency of $200 \mathrm{~Hz}$. The authors assessed the accuracy of the set GPS/IMU at $30 \mathrm{~cm}$. The quality was improved to the level of a few centimeters thanks to the CCD camera images.

In the case presented in (Wallace et al., 2012) the results were obtained with a decimeter accuracy. The authors pointed to the inadequately designed control points as the main cause (too large) and the systematic sampling properties of the laser scanner. Beam divergence, which is high in Ibeo LUX laser scanner, affects predominantly the horizontal accuracy of the point cloud in the direction along the track. The aim of the research regarding forest areas is not without significance in this respect.

In (Glennie et al., 2013), the same scanner was used as in (Wallace et al., 2012) Ibeo LUX, which has the accuracy of only $10 \mathrm{~cm}$. The accuracy of the study results was assessed for the following heights: $6 \mathrm{~cm}$ to $15 \mathrm{~cm}$, and topographically at the level of $20 \mathrm{~cm}$.

The study (Eisenbeiss et al., 2009) analyzes the accuracy of the trajectory of the moving UAV. Surveying reference measurement was used, which was carried out with a precision total station measuring $360^{\circ}$ prism, installed in the UAV. However, the experiment, which was very reliably planned and carried out, has a fundamental drawback. The UAS prototype was equipped with a single-freqency GPS module manufactured by U-blox.

Currently, also commercial solutions of leading companies in the market of laser scanners and satellite receivers are available. There are considerable differences in the UAS as well. One such solution has a rotor span of almost $3 \mathrm{~m}$ and $50 \mathrm{~kg}$ payload capacity. It is highly probable that the advantages of this solution compared to the ALS (LiDAR) is only limited to the lack of staff in the UAV. In the UAV which is so large, it is surprising that it is equipped only in a single-frequency GNSS receiver with precision of the coordinates in real time at the level of $20 \mathrm{~cm}$. In another solution, as claimed by the manufacturer, the set IMU/GNSS is characterized by the position accuracy at $0.05 \mathrm{~m}-0.3 \mathrm{~m}$. On the other hand, laser scanner is available in three types, differing in range and accuracy. For the most accurate variant, the manufacturer claims the accuracy to be at the level of $5 \mathrm{~mm}$ for the maximum range of up to $420 \mathrm{~m}$. The minimum range is also significant, in this case it is $1.2 \mathrm{~m}$. This is the distance which the measured object may be approached to.

The analysis of the source literature proves that it is advisable to complement the laser scanner measurement with CCD camera images. In some cases, it may serve to increase the accuracy of the study results by using accurate georeferencing of the images.

The accuracy of the results obtained from the UAS may not be the highest possible to achieve for a specific object by surveying methods, but a compromise between the accuracy and the economic side of measurements may point to the UAS as more advantageous. Also, specific conditions of place and measurements can significantly limit, or exclude, other surveying techniques.

The technical parameters provided by the manufacturers of mass-produced equipment, look very promising as to the possibilities of using laser scanning with the UAS in engineering surveying. It will be possible to attempt to carry out measurements of the shape, displacements and deformations of various engineering structures, including industrial structures. 


\section{Summary}

The presented examples illustrate only a part of the possible applications of laser scanning, but they demonstrate how useful this technology has become in virtually all issues related to engineering surveying and related applications. The most often used scanning methods include terrestrial methods, i.e. stationary and mobile which, depending on the structure, may substantially differ in the arrangement of stands, ground control points, or measurement platform (mobile solutions). The scanning carried out using UAVs looks promising. After eliminating temporary problems with the accuracy of data acquisition and the duration of the flight, it will facilitate the access to additional information about the geometry of structures, unachievable from the ground stations. Measurement methods, in spite of their rapid development, tend to be more refined than the current method for developing point clouds. Data processing algorithms associated with filtration, extraction and data modeling, represent a very extensive subject. Modeling can be performed based on twodimensional sections, three-dimensional spatial axes, simple geometric solids, and even sophisticated surfaces imaging local deformations of the structure. The condition for economic and correct modeling is, however, appropriate filtration and extraction of data, in which automation is probably the biggest challenge in the field of processing data from laser scanning.

\section{Acknowledgements}

The paper was prepared within the scope of the AGH University of Science and Technology statutory research no. 11.11.150.005 in 2016

\section{References}

Draeyer, B., \& Strecha, Ch., (2014). Pix4D White paper- How accurate are UAV surveying methods? https://support.pix4d.com/hc/en-us/sections/200591029Scientific-White-Papers-and-Presentations\#gsc.tab=0

Eisenbeiss, H., Stempfhuber, W., \& Kolb, M., (2009). Genauigkeitsanalyse der 3DTrajektorie von Mini-UAVs. In: Zukunft mit Tradition "29. WissenschaftlichTechnische Jahrestagung der DGPF", Ed.: Seyfert, E., Publikationen der Deutschen Gesellschaft für Photogrammetrie, Fernerkundung und Geoinformation (DGPF) e.V., Potsdam, 407-417

Gikas, V. (2012). Three-dimensional laser scanning for geometry documentation and construction management of highway tunnels during excavation. Sensors, 12 (8), $11249-11270$

Glennie, C., (2007). Rigorous 3D error analysis of kinematic scanning LIDAR systems. Journal of Applied Geodesy, 1 (2007), 147-157. de Gruyter, doi: 10.1515/JAG

Glennie, C., Brooks B., Ericksen, T., Hauser, D., Hudnut, K., Foster, J., \& Avery, J., (2013). Compact Multipurpose Mobile Laser Scanning System — Initial Tests and Results. Remote Sensing, 5, 521-538. doi:10.3390/rs5020521

Gocał, J. (2010). Geodezja inżynieryjno-przemysłowa, część 3. ISBN: 978-83-7464327-6, Wydawnictwa AGH, Kraków

Hoult, N.A. \& Soga, K. (2014). 11 - Sensing solutions for assessing and monitoring tunnels, In Woodhead Publishing Series in Electronic and Optical Materials, edited by M.L. Wang, J.P. Lynch and H. Sohn, Woodhead Publishing, Volume 56, 309-346, Sensor Technologies for Civil Infrastructures 
Huber, P, J. (2009). Robust Statistics. ISBN 978-0-470-12990-6. John Wiley \& Sons Inc. Hoboken, New Jersey

Kiciak, P. (2005). Podstawy modelowania krzywych i powierzchni. WNT, Warszawa

Lefsky, M.A., Cohen W.B., Parker, G.G., \& Harding, D.J. (2002). Lidar Remote Sensing for Ecosystem Studies. BioScience, vol. 52 (1), 19-30.

Lenda G., Ligas M., Lewińska P., Szafarczyk A., (2016). The use of surface interpolation methods for landslides monitoring, KSCE Journal of Civil Engineering, vol. 20, ISSN 1226-7988

Lenda, G., Marmol, U., Mirek, G. (2015). Accuracy of laser scanners for measuring surfaces made of synthetic materials. Photogrammetrie Fernerkundung Geoinformation ; ISSN 1432-8364. Issue 2015 H. 5, 357-372

Li, Z., Zhu, C., Gold, C.(2004). Digital Terrain Modeling: Principles and Methodology. ISBN 9780415324625. CRC Press, New York

Nagai, M., Chen, T., Ahmed, A., \& Shibasaki, R., (2008). UAV Borne Mapping by Multi Sensor Integration. The International Archives of the Photogrammetry, Remote Sensing and Spatial Information Sciences. Vol. XXXVII. Part B1. Beijing

Novaković, G., Lazar A., Kovačič S. \& Vulić M. (2014). The Usability of Terrestrial 3D Laser Scanning Technology for Tunnel Clearance Analysis Application. Applied Mechanics and Materials, vol. 683, 219-224. Trans Tech Publications

Olea, R.A., (1997). Geostatistics for engineers and earth scientists. Kluwer Academic Publishers.

Pejić, M. (2013). Design and optimisation of laser scanning for tunnels geometry inspection. Tunnelling and Underground Space Technology, Volume 37, 199-206

Pilecki R., (2012). Zastosowania naziemnego skanera laserowego. Mechanika, czasopismo techniczne, wyd. Politechnika Krakowska.

Ries L., (1993). Areas of influence for IDW-interpolation with isotropic environmental data, Catena, Vol. 20, No.1-2, DOI: 10.1016/0341-8162(93)90039-R

Scaioni, M., Longoni, L., Melillo, V., \& Papini, M. (2014). Remote Sensing for Landslide Investigations: An Overview of Recent Achievements and Perspectives. Remote Sensing, 6(10), 9600-9652. doi:10.3390/rs6109600

Siddiqi, K., Pizer, S.M. (2008). Medial Representations: Mathematics, Algorithms and Applications. ISBN: 978-1-4020-8657-1. Springer

Strach, M. (2013). Nowoczesne techniki pomiarowe w procesie modernizacji i diagnostyce geometrii torów kolejowych (Modern surveying techniques in the process of modernization and diagnostics of railway track geometry). Rozprawy, Monografie (Dissertations, Monographs) ISBN 978-83-7464-618-5. Wydawnictwa AGH, Krakow

Strurzengger M., Yan M., Stead D., Elmo D., (2007). Application and limitations of ground-based laser scanning in rock slope characterization. Rock Mechanics: Meeting Society's Callenges and Demands, London

Tong X., Liu X., Chen P., Liu S., Luan K., Li L., Liu S., Liu X., Xie H., Jin Y. \& Hong Z. (2015). Integration of UAV-Based Photogrammetry and Terrestrial Laser Scanning for the Three-Dimensional Mapping and Monitoring of Open-Pit Mine Areas, Remote Sens. vol. 7(6), 6635-6662. doi:10.3390/rs70606635

Wallace, L., Lucieer, A., Watson, Ch., \& Turner, D., (2012). Development of a UAVLiDAR System with Application to Forest Inventory. Remote Sensing, 4, 15191543. doi:10.3390/rs4061519

Weixing, W., Weisen, Z., Lingxiao, H., Vivian, V. \& Zhiwei, W. (2014). Applications of terrestrial laser scanning for tunnels: a review, Journal of Traffic and Transportation Engineering (English Edition), Volume 1, Issue 5, 325-337 
Wirth, H. (2008). Der neue Lichtraummesszug LIMEZ III der Deutschen Bahn AG. Zeitschrift für Geodäsie, Geoinformation und Landmanagement 133 (3), 180-186

Yang, B. \& Fang, L. (2014). Automated Extraction of 3-D Railway Tracks from Mobile Laser Scanning Point Cloud, IEEE Journal of Selected Topics and Applied Earth Observations and Remote Sensing

Zhang, C., Arditi, D. \& Chen, Z. (2013). Documentation and visualization of an as built tunnel by combining $3 \mathrm{D}$ laser scanning and web mapping, ISPRS International Archives of the Photogrammetry, Remote Sensing and Spatial Information Sciences; vol. XL-2/W2, 139-144. DOI: 10.5194/isprsarchives-XL-2W2-139-2013

Authors:

Grzegorz Lenda 1),Ph.D, grzenda@agh.edu.pl

Andrzej Uznański ${ }^{1)}$, Ph.D, auznan@agh.edu.pl

Michał Strach ${ }^{1)}$, D.Sc, Eng., strach@agh.edu.pl

Paulina Lewińska ${ }^{1)}$, Ph.D, lewinska.paulina@gmail.com

1) AGH University of Science and Technology

Faculty of Mining Surveying and Environmental Engineering

Department of Engineering Surveying and Civil Engineering, 30 Mickiewicza Av., $30-059$ Krakow, Poland 